\title{
The Northeast Scotland's 5-year TEVAR Experience
}

\author{
Yen Ming Chan ${ }^{*}$, Paul Bachoo ${ }^{2}$, Ben Cooper ${ }^{3}$, Joanna Janczyk ${ }^{4}$, Bryce Renwick ${ }^{2}$ \\ ${ }^{1}$ Vascular Surgery Department, Aberdeen Royal Infirmary, NHS Grampian, UK \\ ${ }^{2}$ Consultant Vascular Surgeon, UK \\ ${ }^{3}$ Vascular Surgery Specialist Nurse, UK \\ ${ }^{4}$ Vascular Surgery Physician Associate, UK
}

*Corresponding Author: Yen Ming Chan, Vascular Surgery Department, Aberdeen Royal Infirmary, NHS Grampian, UK, Email: sharonyenming.chan@nhs.net

\begin{abstract}
Background and Aims: Thoracic Endovascular Repair (TEVAR) is the preferred option for managing aneurysm disease of the Descending Thoracic Aorta (DTAA) and complications of acute Thoracic Aortic Dissection (TAD). We describe our early Northeast Scotland 5-year experience of TEVAR.

Methods: Retrospective analysis of all emergency and elective TEVAR from January 2012 - July 2017 was performed. Patient demographics, presentation, imaging, operative details, survival status and surveillance CTA status were recorded. Technical success was defined as exclusion of the DTAA without Type 1 endoleak or conversion to open repair or sealing of the proximal entry in acute dissection.

Results: We identified 10 (M: F ratio of 4:1, mean age of 67 years old; standard deviation 17 years) with a technical success of 100\%. 7(70\%) cases were performed as emergency (3 symptomatic dissection, 1 rupture dissection, 1 rupture aneurysm, 1 aortic transection, 1 rupture penetrating aortic ulcer). Postoperative SCI was not observed. 1 (10\%) patient had prophylactic spinal cerebrovascular fluid (CSF) drainage. 3 (30\%) patient had coverage of the left subclavian artery (LSA). Mean follow-up was 12 months.30-day mortality was zero, mortality at follow up was $10 \%(n=1)$. In hospital, post-operative morbidity was 30\%. Endoleak was noted in 2 (20\%) patients at follow up.
\end{abstract}

Conclusion: This study demonstrates the relatively high incidence of unscheduled admission and treatment for this pathology and encouraging early results. Prophylactic measure for SCI remains a topic for debate.

Keywords: Thoracic aortic aneurysm, thoracic aortic dissection, thoracic aorta endovascular repair

Abbreviations: TEVAR: Thoracic Endovascular Aortic Repair, DTA: Descending Thoracic Aorta, DTAA: Descending Thoracic Aortic Aneurysm, TAD: Thoracic Aortic Dissection, SCI: Spinal Cord Ischemia, CTA: Computed Tomography Angiography, LSA: Left Subclavian Artery, CSF: Cerebrospinal Fluid, CTAG: Conformable Gore ${ }^{\circledR}$ TAG ${ }^{\circledR}$ Thoracic Endoprosthesis, ESVS: European Society for Vascular Surgery

\section{INTRODUCTION}

Descending Thoracic Aorta (DTA) diseases represent a spectrum of pathology ranging from degenerative to traumatic. [1] Epidemiological data on DTA disease are limited and there is no report on the exact overall incidence. Often DTA disease remains undiagnosed and wrongly attributed to other acute conditions such as cardiac arrest or myocardial infarction. [2]

Historically, open surgical repair has been the mainstay treatment for DTA disease. Although it confers favourable long term outcome; morbidity and mortality remains high. [3,4] Since Dake first description of the Thoracic Endovascular Repair (TEVAR) in 1994, there has been a shift in practice towards this minimally invasive alternative, which has a better morbidity and mortality profile. This treatment paradigm is reflected in the guidelines published by the European Society for Vascular Surgery (ESVS). [1]

Despite high technical success and good patient related outcomes, TEVAR is not risk-free. Of all complications, spinal cord ischemia (SCI) after TEVAR is most devastating and its prevention and management remain significant areas of on-going research and debate. The incidence of paraplegia or paraparesis as a consequence has been reported to range from $2.5 \%$ to $8 \%$. [5-7] Extensive coverage of the 
thoracic aorta, occlusion of the Left Subclavian Artery (LSA) or Hypo gastric Artery and prolonged hypotension are some of the risk factors associated with postoperative spinal ischemia. Some authors use spinal drainage in high risk patients but data shows no significant benefit in support of routinely using this technique. [7,8] Others recommend routine revascularisation of the LSA if it is to be covered by an endograft-[6,9]

Our unit is a tertiary trauma centre in the northeast Scotland and serves a population of approximately 525,000. We started performing TEVAR in 2012 for both elective and emergency cases. This is a descriptive retrospective study reviewing our 5-year TEVAR experience and outcomes.

\section{METHODS}

A retrospective analysis of all patients undergoing Thoracic Endovascular Aortic Repair in Aberdeen Royal Infirmary from January 2012 - July 2017 were included. In all cases, the operating team consisted of a Vascular Surgeon and Interventional Radiologist. Conformable Gore ${ }^{\circledR}$ TAG ${ }^{\circledR}$ Thoracic Endoprosthesis (CTAG) device (W. L. Gore and Associates) was used in all cases. Patients with Stanford Type A thoracic aortic

Table1. Details of all TEVAR cases including clinical presentation, complications, follow-up CTA and survival

\begin{tabular}{|c|c|c|c|c|c|c|c|c|}
\hline \multirow{2}{*}{$\begin{array}{l}\text { Patient } \\
\text { demographic } \\
\text { (age/gender) }\end{array}$} & \multirow{2}{*}{$\begin{array}{l}\text { Clinical } \\
\text { Presentation }\end{array}$} & \multirow[t]{2}{*}{ Pathology } & \multicolumn{3}{|l|}{ Complications } & \multicolumn{2}{|c|}{ Follow-up CTA } & \multirow[t]{2}{*}{ Survival } \\
\hline & & & peri-operative & 30-day & SCI & Months & Endoleak & \\
\hline $65 \mathrm{M}$ & Emergency & $\begin{array}{l}\text { Symptomatic Thoracic } \\
\text { Aortic dissection }\end{array}$ & Nil & $\begin{array}{l}\text { Acute } \\
\text { Renal } \\
\text { Failure } \\
\end{array}$ & No & 0 & No & Alive \\
\hline $75 \mathrm{M}$ & Elective & $\begin{array}{l}\text { Thoracic Aortic } \\
\text { Aneurysm }\end{array}$ & Nil & Nil & No & 30 & $\begin{array}{|ll|}\text { Type } 1 & 1 \\
\text { conservative }\end{array}$ & Alive \\
\hline $75 \mathrm{M}$ & Elective & $\begin{array}{l}\text { Thoracic Aortic } \\
\text { Aneurysm }\end{array}$ & $\begin{array}{l}\text { Rupture of EIA } \\
\text { (repaired with } \\
\text { interposition } \\
\text { graft) }\end{array}$ & \begin{tabular}{|l|} 
Wound \\
dehiscence \\
(from \\
rupture EIA \\
repair)
\end{tabular} & No & 49 & No & Alive \\
\hline $45 \mathrm{~F}$ & Emergency & $\begin{array}{l}\text { Traumatic Aortic } \\
\text { transection }\end{array}$ & Nil & Nil & No & 3 & Type IV & Dead \\
\hline $76 \mathrm{M}$ & Elective & $\begin{array}{l}\text { Thoracic Aortic } \\
\text { aneurysm associated } \\
\text { with ulcer }\end{array}$ & Nil & Nil & No & 25 & No & Alive \\
\hline $87 \mathrm{~F}$ & Emergency & $\begin{array}{l}\text { Rupture Thoracic } \\
\text { Aortic Aneurysm }\end{array}$ & Nil & Nil & No & 18 & No & Alive \\
\hline $77 \mathrm{M}$ & Emergency & $\begin{array}{l}\text { Symptomatic Thoracic } \\
\text { Aortic Dissection }\end{array}$ & Nil & Pneumonia & No & 0 & No & Alive \\
\hline $37 \mathrm{M}$ & Emergency & $\begin{array}{l}\text { Rupture Thoracic } \\
\text { Aortic Dissection }\end{array}$ & Nil & Nil & No & 0 & No & Alive \\
\hline $60 \mathrm{M}$ & Emergency & $\begin{array}{l}\text { Symptomatic Thoracic } \\
\text { Aortic Dissection }\end{array}$ & Nil & Nil & No & 0 & No & Alive \\
\hline $83 \mathrm{M}$ & Emergency & $\begin{array}{l}\text { Rupture Thoracic } \\
\text { Aorta Penetrating } \\
\text { Ulcer }\end{array}$ & Nil & Nil & No & 0 & No & Alive \\
\hline
\end{tabular}

CTA - Computed Tomography Angiography; SCI - Spinal Cord Ischemia; EIA - external iliac artery pathology were excluded from this study. Patient demographics, clinical details, survival, presentation, imaging, operative records and information on follow-up were obtained from web-based hospital information system (Track care) and Radiology Information System (Picture Archiving and Communication System - PACS). Technical success was defined when TEVAR was performed without conversion to open surgical and no endoleak on completion angiogram was observed. The last date of Computed Tomography Angiography (CTA) was documented as the last follow-up date. Data obtained were recorded on a Microsoft Excel sheet and analysed using the statistical package offered by the programme.

\section{Results}

A total of 10 TEVAR were performed in the study period. $8(80 \%)$ were male, $2(20 \%)$ were females. The mean age was 67 years old (range 37 - 87years). 7 (70\%) were carried out as an emergency. Figure 1 (Fig 1) demonstrates the classification of pathology based on clinical presentation. Technical success rate was $100 \%$. All cases were performed under general anaesthetic with post-operative admission to High Dependency Unit. Table 1 summarises all cases and their outcome. 


\section{Distribution of Pathology based on Clinical Presentation}

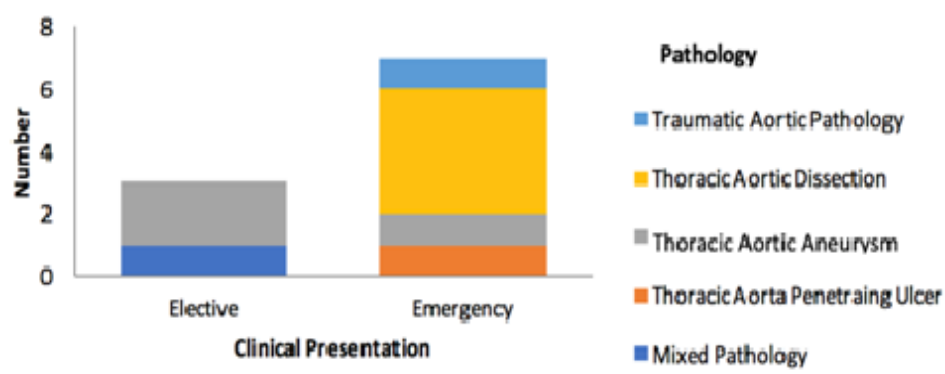

Figure1. Classification of thoracic Aortic pathology based on clinical presentation

Three $(30 \%)$ of our patients had coverage of the LSA. One of these patients had spinal CSF drainage. Neither of the patients had revascularisation of LSA. None of the patients developed spinal cord ischemia. There was 1 (10\%) intra-operative complication. This was an external iliac artery wire injury, which was repaired with an open interposition graft in the same setting. Post-operative complications were $30 \%$. These complications were wound dehiscence from the additional procedure performed following a peri-operative injury to an artery, acute renal failure and pneumonia. The mean follow-up was 12.9 months (388 days) and range from $0-50$ months $(0-1499$ days). On follow-up CTA, there were 2 endoleak (1 type I, 1 type IV). Decision for conservative management due to static size of aneurysm sac for the patient with type I endoleak. There were no cases of stent migration or occlusion of stents on follow-up.

Mortality was $1(10 \%)$ and there was no 30-day mortality. Cause of death was due to major haemorrhage from Type IV endoleak with fistulation to oesophagus 3-month postprocedure.

\section{DISCUSSION}

We recently started performing TEVAR in our unit. Therefore, we consider it useful to conduct this review of our experience to contribute to current literature regarding the efficacy of TEVAR and assess our outcomes compared to other centres.

Our findings show that DTA diseases has a male predominance $(80 \%)$ with a mean age of 67 years old, which is in keeping with other studies.[4,10-12] We performed two thirds of our cases in an emergency setting, which is similar to some of the bigger series. $[10,13]$ Our emergent cases were due to symptomatic dissection, rupture of the underlying aortic pathology and one case of traumatic aortic transection. These distributions of pathology were also found to be comparable with the bigger series. In contrast, we have a small number of elective cases. All of which were due to degenerative aneurysm diagnosed incidentally during investigation for unrelated clinical symptoms.

We used Conformable Gore ${ }^{\circledR}$ TAG ${ }^{\circledR}$ Thoracic End prosthesis (CTAG) device in all cases as it provides better conformability, resistance to compression and good range of sizes that allows applicability in a wide spectrum of DTA pathology. [14] We achieved a 100\% technical success rate with no conversion to open surgical repair. This result may be explained by our institution's practice of combined input from both vascular surgeons and interventional radiologist in all cases. However, we had one case of peri-operative vessel complication that required one additional procedure, which was performed in the same setting. We appreciate that this finding may be bias due to our small cohort group similar to that reported by Neo et al. [11]

Of our post-operative complications, none led to a need for further intervention or escalation of care. The patient with pneumonia improved with antibiotics whilst the patient with wound dehiscence was treated conservatively. Meanwhile, the patient who developed acute renal failure had thrombus affecting the renal vessel in his primary pathology and as such is not primarily due to TEVAR. Our series demonstrates a morbidity profile that is slightly higher in comparison to Neo et al that reported a $22.2 \%$ major post-op complication in their small cohort study group. [11] However, we had no cardiac complications. A meta-analysis reported that the incidence of cardiac complication can be as high as $14.6 \%$ and overall major complication including all other systemic ischemia was $41.4 \%$. [4]Although spinal CSF drainage is not routinely performed and none of 
the three patients with LSA coverage in our series had revascularisation of LSA, our SCI rate is $0 \%$. Therefore, we cannot conclude if either of these prophylactic measures influence our SCI rate as suggested by Hnath et al and Both at al in their studies.[6,15] Meanwhile, Scott and colleagues [16]advocate that maintenance of norm tension peri- and postoperatively is critical in preventing SCI. In addition, they highlighted the importance of regular monitoring and assessment for clinical signs of SCI which should prompt early prophylactic measures.

Two (20\%) of our patients had endoleak on follow-up which was within the range of 9 $38 \%$ reported in literature. [10-13,17,18] None of our endoleak requires intervention although the only mortality in our series was associated with a type IV endoleak. We did not report any stent migrations, thrombosis or fracture. We report 30-day mortality of $0 \%$ despite having a higher proportion of cases performed in an emergency setting. Most studies showed higher rate of 30-day mortality in emergency group compared to elective with a rate as high as $28 \%$ in their degenerative aneurysm group by Leurs et al. $[10,12]$

We acknowledge that there are several weaknesses to this study. Firstly, our cohort size is small which makes interpretation of results biased. In addition, this is a retrospective review with variable length of follow-up. The variability of follow-up included cases, which has less than 1-year follow up which may mean that some of the outcome have yet to be known.

\section{CONCLUSION}

We perform more emergency TEVAR cases with better 30-day mortality rates and morbidity rates similar to that in literature. Our experience supports the trend towards this minimally invasive alternative in managing DTA diseases particularly in the acute setting. Meanwhile, prophylactic measures for SCI remain a topic of debate and an area for research.

\section{REFERENCES}

[1] Committee, W. et al. Editor's Choice Management of Descending Thoracic Aorta Diseases: Clinical Practice Guidelines of the European Society for Vascular Surgery(ESVS). Eur. J. Vasc. Endovasc. Surg.53, 4-52(2017).

[2] Hiratzka, L. F. et al. 2010 ACCF/AHA/AATS/ ACR/ASA/SCA/SCAI/SIR/STS/SVM

Guidelines for the Diagnosis and Management of Patients With Thoracic Aortic Disease. Journal of the American College of Cardiology 55, (2010).
[3] Lee, W.-Y. et al. Outcomes of Open Surgical Repair of Descending Thoracic Aortic Disease. Korean J. Thorac. Cardiovasc. Surg.47, 255261 (2014).

[4] Cheng, D. et al. Endovascular Aortic Repair Versus Open Surgical Repair for Descending Thoracic Aortic Disease. A Systematic Review and Meta-Analysis of Comparative Studies. $J$. Am. Coll. Cardiol.55, 986-1001 (2010).

[5] Jonker, F. H. W. et al. Open surgery versus endovascular repair of ruptured thoracic aortic aneurysms. J. Vasc. Surg.53, 1210-1216 (2011).

[6] Buth, J. et al. Neurologic complications associated with endovascular repair of thoracic aortic pathology: Incidence and risk factors. a study from the European Collaborators on Stent/Graft Techniques for Aortic Aneurysm Repair (EUROSTAR) registry. J. Vasc. Surg. 46, 1101-1103 (2007).

[7] Drinkwater, S. L. et al. The incidence of spinal cord ischaemia following thoracic and thoracoabdominal aortic endovascular intervention. Eur. J. Vasc. Endovasc. Surg.40, 729-735 (2010).

[8] Ullery, B. W. et al. Risk factors, outcomes, and clinical manifestations of spinal cord ischemia following thoracic endovascular aortic repair. $J$. Vasc. Surg. Off. Publ. Soc. Vasc. Surg. [and] Int. Soc. Cardiovasc. Surgery, North Am. Chapter54, 677-84 (2011).

[9] Peterson, B. G., Eskandari, M. K., Gleason, T. G. \& Morasch, M. D. Utility of left subclavian artery revascularization in association with endoluminal repair of acute and chronic thoracic aortic pathology. J. Vasc. Surg.43, 433-439 (2006).

[10] Leurs, L. J. et al. Endovascular treatment of thoracic aortic diseases: Combined experience from the EUROSTAR and United Kingdom Thoracic Endograft registries. J. Vasc. Surg.40, 670-679 (2004).

[11] Neo, W. T., Pua, U. \& Wong, D. E. Thoracic endovascular aortic repair: a local single institution experience. Ann. Acad. Med. Singapore40, 414-7 (2011).

[12] Etezadi, V. et al. Endovascular treatment of descending thoracic aortic disease: singlecenter, 15-year experience. J. Vasc. Interv. Radiol.23, 468-475 (2012).

[13] Ziza, V. et al. Thoracic endovascular aortic repair: A single center's 15-year experience. $J$. Thorac. Cardiovasc. Surg.151, 1595-1603.e7 (2016).

[14] Jordan, W. D. et al. Results of a prospective multicenter trial of CTAG thoracic endograft. in Journal of Vascular Surgery61, 589-595 (2015). 
[15] Hnath, J. C. et al. Strategies to improve spinal cord ischemia in endovascular thoracic aortic repair: Outcomes of a prospective cerebrospinal fluid drainage protocol. J. Vasc. Surg.48, 836840 (2008).

[16] Scott, D. A. \& Denton, M. J. Spinal cord protection in aortic endovascular surgery. $\mathrm{Br} . \mathrm{J}$. Anaesth.117, ii26-ii31 (2016).
[17] Matsumura, J. S. et al. International controlled clinical trial of thoracic endovascular aneurysm repair with the Zenith TX2 endovascular graft: 1-year results. J. Vasc. Surg.47, (2008).

[18] Fairman, R. M. et al. Pivotal results of the Medtronic Vascular Talent Thoracic Stent Graft System: the VALOR trial. J. Vasc. Surg. Off. Publ. Soc. Vasc. Surg. [and] Int. Soc. Cardiovasc. Surgery, North Am. Chapter48, 546-554 (2008).

Citation: Yen Ming Chan, Paul Bachoo, Ben Cooper, Joanna Janczyk, Bryce Renwick. The Northeast Scotland's 5-year TEVAR Experience. ARC Journal of Anesthesiology. 2017; 2(3):5-9. doi: dx.doi.org/10.20431/2455-9792.0203003.

Copyright: () 2017 Authors. This is an open-access article distributed under the terms of the Creative Commons Attribution License, which permits unrestricted use, distribution, and reproduction in any medium, provided the original author and source are credited. 